\title{
Dynamic PET Denoising with HYPR Processing
}

\author{
Bradley T. Christian ${ }^{1,2}$, Nicholas T. Vandehey ${ }^{1}$, John M. Floberg ${ }^{1}$, and Charles A. Mistretta ${ }^{1,3}$
}

${ }^{1}$ Department of Medical Physics, University of Wisconsin-Madison, Madison, Wisconsin; ${ }^{2}$ Department of Psychiatry, University of Wisconsin-Madison, Madison, Wisconsin; and ${ }^{3}$ Department of Radiology, University of Wisconsin-Madison, Madison, Wisconsin

HighlY constrained backPRojection (HYPR) is a promising image-processing strategy with widespread application in time-resolved MRI that is also well suited for PET applications requiring time series data. The HYPR technique involves the creation of a composite image from the entire time series. The individual time frames then provide the basis for weighting matrices of the composite. The signal-to-noise ratio (SNR) of the individual time frames can be dramatically improved using the high SNR of the composite image. In this study, we introduced the modified HYPR algorithm (the HYPR method constraining the backprojections to local regions of interest [HYPR-LR]) for the processing of dynamic PET studies. We demonstrated the performance of HYPR-LR in phantom, small-animal, and human studies using qualitative, semiquantitative, and quantitative comparisons. The results demonstrate that significant improvements in SNR can be realized in the PET time series, particularly for voxel-based analysis, without sacrificing spatial resolution. HYPR-LR processing holds great potential in nuclear medicine imaging for all applications with low SNR in dynamic scans, including for the generation of voxel-based parametric images and visualization of rapid radiotracer uptake and distribution.

Key Words: instrumentation; molecular imaging; PET; denoising; dynamic positron emission tomography; parametric images

J Nucl Med 2010; 51:1147-1154

DOI: 10.2967/jnumed.109.073999

$\mathbf{P}_{1}$ ET is a powerful in vivo imaging modality for tracking dynamic changes in physiologic processes. However, the temporal resolution and, in turn, the underlying physiologic information are often sacrificed by integrating the data collection across time to yield images with adequate signalto-noise ratios (SNRs). Such has been the strategy for ${ }^{18} \mathrm{~F}-\mathrm{FDG}$, the mainstay of PET, using imaging acquisition methods that require only 1 time point to obtain an approximation of glucose metabolism (1). Despite the experimental ease of single-time-point assays, investigators have acknowledged the advantages of acquiring radiotracer time

Received Dec. 16, 2009; revision accepted Mar. 4, 2010.

For correspondence or reprints contact: Bradley T. Christian, Rm. T235, Waisman Center, 1500 Highland Ave., University of WisconsinMadison, Madison, WI 53705

E-mail: bchristian@wisc.edu

COPYRIGHT @ 2010 by the Society of Nuclear Medicine, Inc. courses for quantitative assessment (2,3). Kinetic analysis is generally recognized as a more informative method of quantifying PET data than using simpler methods such as standardized uptake values (4-6) but remains underutilized in the clinical setting in part because of the low SNR accompanying time series data and lengthier acquisition.

Strategies have been explored since the infancy of PET to optimize both data acquisition and algorithm implementation for parameter estimation from dynamic time series (7-9). Preprocessing algorithms (i.e., on sinograms) were developed to exploit the spatiotemporal correlation in dynamic PET scans, with the aim of improving physiologic parameter estimation $(10,11)$. Most strategies have focused on postprocessing algorithms with time series data reconstructed with filtered backprojection (FBP) or ordered-subset expectation maximization. For example, wavelet processing has demonstrated some success as a denoising method on voxelbased time-activity data (12-15). However, wavelet processing is computationally intensive and requires further investigation to optimize the denoising of wavelet coefficients. Methods using spatial constraint algorithms have also been applied to the process of parameter estimation and have yielded measurable reductions in parameter estimation variability (16), but these methods are limited to the spatial constraint of a single time frame and do not take advantage of the increased SNR of the entire time series.

HighlY constrained back PRojection (HYPR) is a promising strategy with widespread application in time-resolved MRI (17) that is also well suited for PET applications requiring time series data. HYPR was initially introduced for the reconstruction of highly undersampled MRI time series data-for example, in applications for which the passage of contrast material through a region is faster than the acquisition capabilities of the MRI system. The HYPR technique involves the creation of a composite image from the entire undersampled radial time series. The individual (undersampled) time frames then provide the high temporal resolution for the weighting matrices, which are multiplied by the composite image containing the high spatial resolution. This technique permits image generation of rapidly changing processes from acquisitions that are radially undersampled by factors of up to several hundred. This method was further refined by constraining the backprojections to local 
regions of interest (ROIs), which was termed HYPR-LR (18). This local reconstruction adaptation of HYPR can accommodate situations in which image sparsity and complete spatiotemporal correlation are not met. For the case of radionuclide imaging studies, in which adequate angular sampling exists during each time frame, the HYPR-LR weighting images are formed by a ratio of convolved versions of the time frame and composite image, thus taking advantage of the spatiotemporal correlations between time frames.

Strategies have been used for the application of partialvolume correction of PET data that use algorithms similar to HYPR-LR. Boussion et al. (19) developed a technique to capture the high spatial resolution from a separate imaging study, such as MRI or CT, using wavelet transforms and applying this information into image-based corrections for the lower-resolution PET (and SPECT) studies. In contrast, the HYPR-LR algorithm captures high SNR by integrating the PET data over the time course of the study but with the same spatial resolution as the original PET data. Filtering functions are then used to obtain high SNR in the independent time frames, without attempting to improve spatial resolution.

HYPR-LR processing holds great potential in nuclear medicine imaging for all applications with low SNR in dynamic scans, including the generation of voxel-based parametric images and visualization of rapid radiotracer uptake and distribution. To evaluate the performance of HYPR-LR postprocessing (i.e., on the FBP-reconstructed dataset), we present data based on qualitative, semiquantitative, and quantitative comparisons in phantom, small-animal, and human studies. For all methods of evaluation, we chose to analyze dynamic measured PET data rather than to use computersimulated data. This method of evaluation was chosen to present an overall performance of HYPR-LR postprocessing using actual studies to demonstrate the practical application of this technique and phantom studies to assess signal fidelity using known concentrations of radionuclide.

\section{MATERIALS AND METHODS}

\section{HYPR-LR Processing}

A schematic of the HYPR-LR method is shown in Figure 1. The general HYPR-LR approach involves the formation of a composite image from several or all of the images in a time series. This image, which provides high-spatial-resolution, high-SNR information, is multiplied by low-spatial-resolution weighting images formed from each time frame. HYPR-LR weighting images are formed by taking a ratio of spatially convolved versions of the time sequence and composite image; thus, each individual frame has an SNR closer to that of the composite. The functional equation for HYPR-LR processing, following the notation of Johnson et al. (18), is:

$$
\mathrm{I}_{\mathrm{H}}=\mathrm{I}_{\mathrm{c}} \cdot \mathrm{I}_{\mathrm{w}}
$$

Eq. 1

where $I_{H}$ is the HYPR image, and $I_{c}$ is the time-averaged composite image. $I_{w}$ is the weighting image derived from the original reconstructed images I; a box-kernel (low-pass) spatial filter function, $\mathrm{F}$; and $\mathrm{I}_{\mathrm{c}}$, given as:

$$
\mathrm{I}_{\mathrm{W}}=\frac{\mathrm{F} \otimes \mathrm{I}}{\mathrm{F} \otimes \mathrm{I}_{\mathrm{c}}}
$$

In the original formulation (18), the $\mathrm{I}_{\mathrm{c}}$ term in the denominator of Equation 2 was represented by a composite formed from a sliding temporal window of the time series (rather than the entire time series). The temporal window for the image, $I_{c}$, was empirically determined by examining the fidelity of the large ROI PET signal (i.e., high SNR) with the HYPR-LR time series of varying window widths. For the studies reported herein, it was found that using the entire time series for this image did not distort the signal fidelity; therefore, the composite image, $I_{c}$, represented the entire summed PET dynamic study - that is,

$$
\mathrm{I}_{\mathrm{c}}=\sum_{\mathrm{i}} \mathrm{PET}_{\mathrm{i}} \cdot \Delta \mathrm{t}_{\mathrm{i}}
$$

Eq. 3

for $\mathrm{i}$ frames of duration $\Delta \mathrm{t}_{\mathrm{i}}$. The filter function used either a 2dimensional (2D; in-plane) or 3-dimensional (3D; volumetric) kernel, F. For dynamic PET studies with time frames of varying duration, the $\mathrm{I}\left(=\mathrm{PET}_{\mathrm{i}}\right)$ images were weighted according to their duration $\left(\Delta t_{i}\right)$. For the HYPR-LR process, the SNR of the individual time frames is largely determined by the SNR of the composite image, and it has been shown that the variance of the HYPR-LR images is inversely proportional to the number of nonzero elements in the filter function (F) (18). However, excessive spatial filtering can also introduce spillover of signals from neighboring regions. A range of spatial filters was investigated to examine this effect.

\section{Experimental}

Evaluating Accuracy and Reduced Voxel SNR. A custom-made miniature Derenzo acrylic phantom (diameter, $40 \mathrm{~mm}$; length, 13 $\mathrm{mm}$ ) was filled with a uniform concentration of aqueous radioactivity $\left({ }^{18} \mathrm{~F}, 1.5 \mathrm{MBq} / \mathrm{mL}\right)$ and scanned using a Concorde microPET $\mathrm{P} 4$ scanner (20). The phantom contained holes (diameters, 0.8, 1.0, $1.25,1.5,2.0$, and $2.5 \mathrm{~mm}$ ) arranged in wedged-shaped groupings, with the center-to-center hole separation being twice the hole
FIGURE 1. Schematic of HYPR-LR processing of dynamic PET data. $4 \mathrm{D}=$ 4-dimensional.

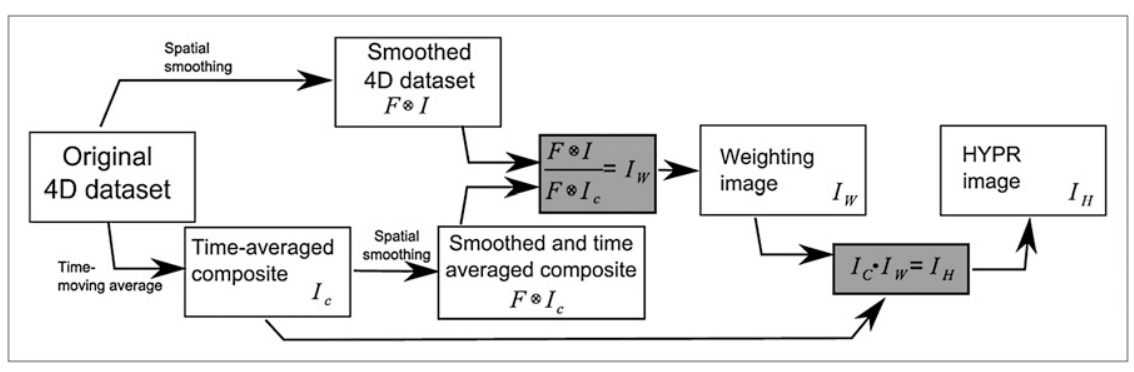


diameter $(21,22)$. Emission and transmission events were binned into 3D sinograms, with a span of 3 and a ring difference of 31, using the system software (version 2.3.3.6). The emission list-mode files were then binned into sixty 1-min frames. For reconstruction, the 3D sinograms were first rebinned to 2 dimensions by the system Fourier rebinning algorithm. The data were reconstructed using FBP with a ramp filter (at the Nyquist frequency cutoff), $a \times 1.5$ image zoom, and no offsets to a matrix size of $128 \times 128 \times 63$ with voxel dimensions of $1.26 \times 1.26 \times 1.21 \mathrm{~mm}$. Corrections for normalization, dead time, random coincidences, attenuation, scatter, and decay of radioactivity were applied using the system software. An in-plane profile through the 2.5 - and $1.5-\mathrm{mm}$ diameter holes was applied to both the FBP and HYPR-LR processed time series. The mean and SD were then calculated for each voxel passing through the profile. The accuracy (bias) was compared on the basis of the profile mean measured concentration, and the SNR was defined as the voxel mean divided by its SD.

Measurement accuracy was also evaluated with a dynamic small-animal PET scan of the rhesus monkey using ${ }^{18} \mathrm{~F}$-fallypride, a PET ligand targeting the dopamine $\mathrm{D}_{2} / \mathrm{D}_{3}$ receptor sites in the brain. ${ }^{18} \mathrm{~F}$-fallypride was administered in 3 injections containing a high-specific-activity radiotracer $(0 \mathrm{~min}, 168 \mathrm{MBq}, 4.2 \mathrm{nmol})$, partially saturating low specific activity (20 min, $189 \mathrm{MBq}, 10.3$ $\mathrm{nmol}$ ), and competing unlabeled blocking dose $(80 \mathrm{~min}, 0 \mathrm{MBq}$, $100 \mathrm{nmol}$ ) into a 5-kg subject, and data were acquired for $160 \mathrm{~min}$. The PET data were binned into dynamic frames of $2 \mathrm{~min}$ per frame. Time-activity curves were generated for the striatum, which demonstrated changing in vivo kinetics throughout the course of the study.

Accuracy in Parameter Estimation. An 80-mm-diameter cylindric phantom with 14-mm internal (cylindric) chambers was scanned using the Concorde microPET P4 scanner. The overall chamber was filled with $16.7 \mathrm{kBq} / \mathrm{mL}$ of ${ }^{18} \mathrm{~F}$ (half-life [ $\mathrm{t}_{1 / 2}$ ], 110 $\min )$; one internal chamber was filled with $26 \mathrm{kBq} / \mathrm{mL}$ of ${ }^{11} \mathrm{C}\left(\mathrm{t}_{1 / 2}\right.$, $20.4 \mathrm{~min}$ ), one chamber with air, and the other with solid acrylic. This phantom was scanned for $40 \mathrm{~min}$ as the ${ }^{11} \mathrm{C}$ activity went from roughly double to half the ${ }^{18} \mathrm{~F}$ background. The acquisition and reconstruction parameters were the same as those described above, except correction for decay was not included in the FBP reconstruction. ROIs (diameter, 9 voxels [11.3 mm], spanning 26 planes, 1,274 total voxels) were placed over the areas containing ${ }^{11} \mathrm{C}$ and ${ }^{18} \mathrm{~F}$ radioactivity. Time-activity curves were generated for each voxel within these regions (voxelwise) and also for averages over the entire region (regionwise). Decay constants from the time-activity curves were estimated using a nonlinear least-squares fit (lsqcurvefit in Matlab [The MathWorks]; TolX $=1 \mathrm{e}-6$, TolFun $=1 \mathrm{e}-6$, MaxIter $=400)$ to the equation $\mathrm{C}(\mathrm{t})=\mathrm{C}_{\mathrm{o}} \mathrm{e}^{-\lambda \mathrm{t}}$, for the parameters of starting concentration $\left(\mathrm{C}_{\mathrm{o}}\right)$ and radionuclide decay constant $(\lambda=$ $\left.\ln (2) / t_{1 / 2}\right)$, with initial guesses for the parameter values fixed to $\pm 50 \%$ of the known values. The accuracy or bias of the estimates was measured by comparing the distribution mean with the known values $\left({ }^{11} \mathrm{C}, 0.034 \mathrm{~min}^{-1} ;{ }^{18} \mathrm{~F}, 0.0063 \mathrm{~min}^{-1}\right)$.

Performance of HYPR-LR in Datasets with High Noise Levels. A human scan of ${ }^{15} \mathrm{O}$-water was acquired, which consisted of sixty 2-s frames initiated with a bolus injection of $2.78 \mathrm{GBq}$ of radiotracer. The scan was acquired using the Advance PET scanner (GE Healthcare) in 2D mode, as specified by the NIH-sponsored Carotid Occlusion Surgery Study. The data were reconstructed using FBP (ramp filter) to a matrix size of $128 \times 128 \times 63$, with voxel dimensions of $2.34 \times 2.34 \times 4.25 \mathrm{~mm}$. HYPR-LR processing was performed using the entire $120 \mathrm{~s}$ of data as the composite image.
Time-activity curves over small and large regions of the brain were compared for accuracy and SNR.

\section{RESULTS}

The results of a semiquantitative comparison of the miniature Derenzo phantom are shown in Figure 2 for the HYPR-LR image using a $3.78 \times 3.78 \times 3.63 \mathrm{~mm}(3 \times 3 \times 3$ voxels) $3 \mathrm{D}$ boxcar smoothing filter. Averaged over the sixty 1-min frames, the mean concentrations at each voxel along the profile are in close agreement, with a mean difference in measured concentration of less than $0.2 \%$ between the FBP and HYPR-LR data. However, the mean coefficient of variation (COV) (SD/mean) is significantly less in the HYPR-LR data, with a COV FBP of $22 \%$ and a COV HYPR of $9 \%$ ( $P<0.00002)$, suggesting a more than 2 -fold improvement in SNR. A profile of 1 voxel (width, $1.26 \mathrm{~mm}$ ) was used for the data in Figure 2. Also shown (far right column) are the results of postprocessing with just the smoothing filter (F). This processing yields a reduced variability, with smooth COV of $13 \%$, but is accompanied by the inability to resolve the $1.5-\mathrm{mm}$ regions and reduced activity recovery in the 2.5-mm regions.

The accuracy of the parameter estimates was examined using a phantom with 2 radioactive sources of different decay constants. The FBP-reconstructed and HYPR-LR-processed images using 1-min time frames are shown in Figure 3. HYPR-LR processing was performed using a $3.78 \times 3.78 \times$ $3.63 \mathrm{~mm}(3 \times 3 \times 3$ voxels $) 3 \mathrm{D}$ boxcar spatial filter $(\mathrm{F})$ on the composite and dynamic images. Volumes of 1,274 voxels $\left(2.4 \mathrm{~cm}^{3}\right)$ were selected from the regions within the ${ }^{11} \mathrm{C}$ and ${ }^{18} \mathrm{~F}$ volumes. The decay constants were compared by regionwise (averaged over all voxels) and voxelwise analysis. Figure 4A displays the regionwise time-activity curves. The decay constants from these time-activity curves were estimated using a nonlinear least-squares fit to the equation $\mathrm{C}(\mathrm{t})=\mathrm{C}_{\mathrm{o}} \mathrm{e}^{-\lambda \mathrm{t}}$, for the parameters of starting concentration $\left(C_{o}\right)$ and radionuclide decay constant $\left(\lambda=\ln (2) / t_{1 / 2}\right)$, with the results shown in Table 1 . For the ${ }^{11} \mathrm{C}$ region, both the FBP and HYPR-LR processing yielded similar values within the true range of the decay constant. For the ${ }^{18} \mathrm{~F}$ region, there was little difference between the FBP and HYPR-LR estimates, regardless of spatial filtering in the HYPR-LR processing. The $2 \%-6 \%$ underestimation from the theoretic value is attributable to using only $40 \mathrm{~min}$ of data to estimate this rate constant with a 110-min half-life.

\section{ROI Estimates of Decay Constant $\left(\mathrm{min}^{-1}\right)$ (Table 1)}

The decay constants were also estimated from the timeactivity curve of each voxel within this region. The distributions of the voxel-based decay constant estimates are shown in Figure 4B. For the native FBP image set, approximately $14 \%$ of the voxels yielded estimates with low or negative decay constants $\left(<0.005 \mathrm{~min}^{-1}\right)$ or high constants $(>0.1$ $\min ^{-1}$ ) because of the high levels of noise in these timeactivity curves. The outliers were not included in the estimation of the mean of $\lambda$ for the FBP data. The parameter 


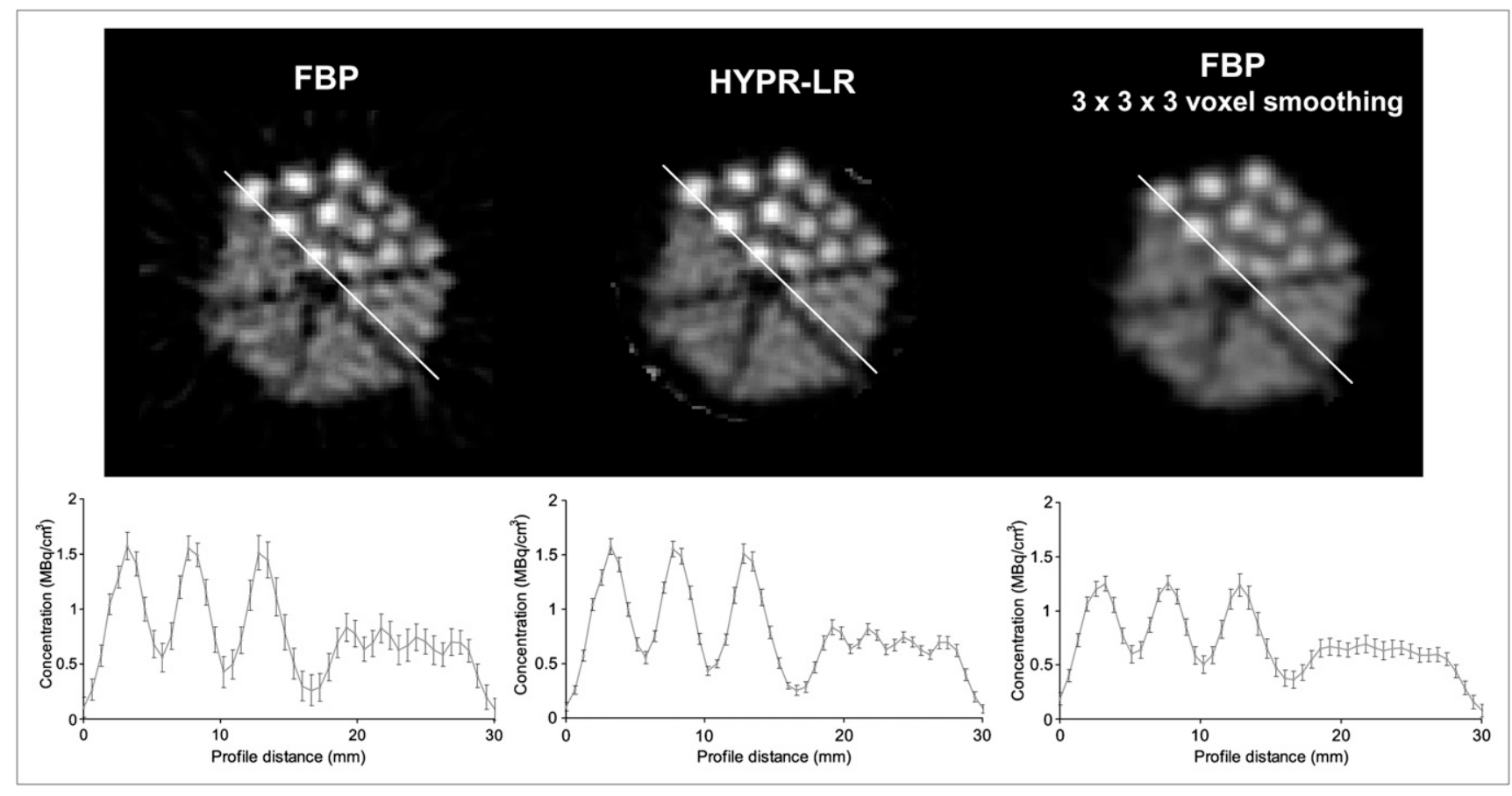

FIGURE 2. HYPR-LR processing of small-animal PET images of miniature Derenzo phantom. Transaxial slices of 1-min frames for FBP, HYPR-LR processing, and $3.78 \times 3.78 \times 3.63 \mathrm{~mm}(3 \times 3 \times 3$ voxels $) 3 \mathrm{D}$ boxcar smoothing of FBP image are displayed on top. Profiles (as shown on the images) plotting mean and SD of 1-min frames over entire 60-min study are shown on bottom. All images are shown using same window and threshold.

estimates agreed closely with the known decay rates and yielded a reduced COV (SD/mean), compared with the FBP processing ( $15 \%$ vs. $76 \%$, respectively), using the HYPR-LR processing. Increased spatial filtering of the weighting image was investigated, and a reduction in the variance in the distribution of parameter estimates was found $(P<0.0001$ for all HYPR-LR data); however, this was at the cost of increased bias due to the mixing of signal from the ${ }^{18} \mathrm{~F}$ background region (Supplemental Fig. 1; supplemental materials are available online only at http://jnm.snmjournals. org).

To examine the effects of rapidly changing temporal kinetics under experimental conditions, HYPR-LR processing was performed on a PET neuroligand study having kinetics that were dramatically altered mid scan. A timeactivity curve of the data is shown in Figure 5. For a voxel ROI placed in the high-uptake region of the striatum, reduced noise is present in the HYPR-LR processed data, with no apparent bias between methods in the time-activity curves. For this study, the composite image consisted of the entire $160 \mathrm{~min}$ of the study, including the period of washout at approximately $80 \mathrm{~min}$, which is dominated by the dissociation constant $\mathrm{k}_{\text {off }}$ rate of fallypride $\left(\sim 0.025 \mathrm{~min}^{-1}\right)$ (23).

Figure 6 displays a comparison of ${ }^{15} \mathrm{O}$-water in the human brain to illustrate the effects of images with high levels of noise. A single composite image was used for HYPR-LR processing of this study, and the individual frames were
FIGURE 3. HYPR-LR processing of images of multiradionuclide phantom. Cylindric volume is filled with ${ }^{11} \mathrm{C}\left(\mathrm{t}_{1 / 2}\right.$, $20.4 \mathrm{~min}$ ), and surrounding volume contains ${ }^{18} \mathrm{~F}\left(\mathrm{t}_{1 / 2}, 110 \mathrm{~min}\right)$. Total summed image (0-40 $\mathrm{min})$ and FBPreconstructed images of 1-min frames at 0,20 , and $40 \mathrm{~min}$ are shown on top. Blurred composite (sum) image and 1min frames of data processed with HYPR-LR using $3.78 \times 3.78 \times 3.63 \mathrm{~mm}$ $(3 \times 3 \times 3$ voxels) $3 \mathrm{D}$ boxcar filter are shown on bottom. All images are scaled to maximum intensity of $37 \mathrm{kBq} / \mathrm{mL}$.

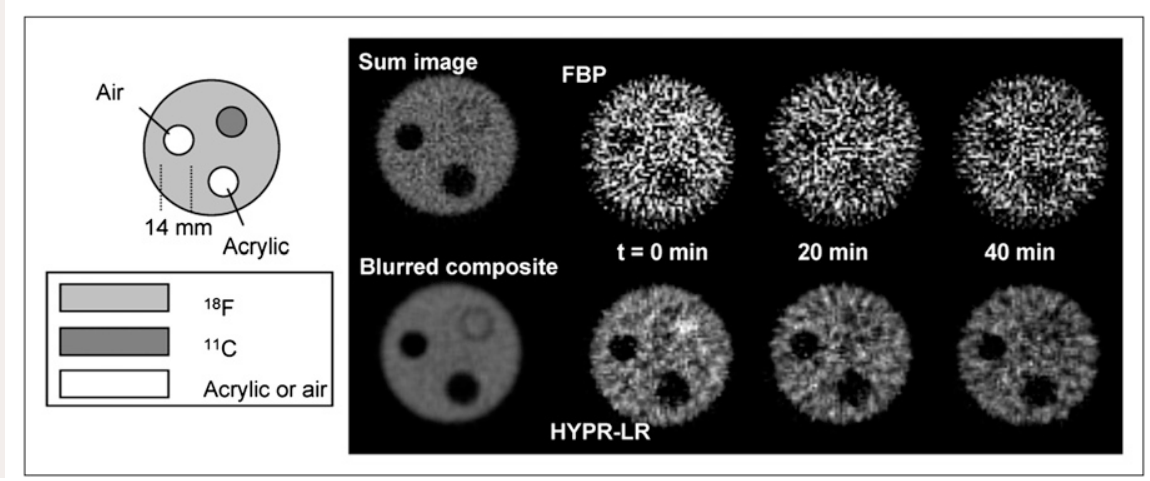



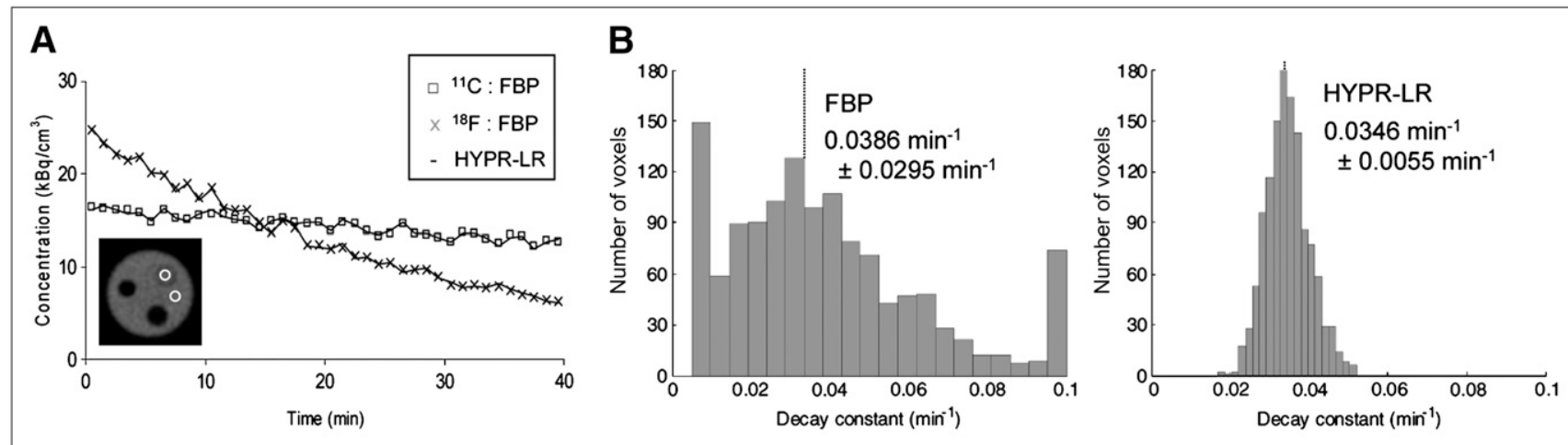

FIGURE 4. Quantitative accuracy of HYPR-LR processing. (A) Regionwise analysis: graph displays time-activity curves of volume containing 1,274 voxels $\left(2.4 \mathrm{~cm}^{3}\right)$ centered over ${ }^{11} \mathrm{C}$ and ${ }^{18} \mathrm{~F}$ areas. When averaged over entire volume, time-activity curves are almost identical for FBP data and HYPR-LR data. (B) Voxelwise analysis: results of voxel-based analysis of measured decay constants from ${ }^{11} \mathrm{C}$ region. HYPR-LR processing was performed using $3.78 \times 3.78 \times 3.63 \mathrm{~mm}(3 \times 3 \times 3 \mathrm{voxels})$ $3 \mathrm{D}$ boxcar filter. Values on each graph indicate mean \pm SD for each distribution. Decay constant for ${ }^{11} \mathrm{C}$ is $0.034 \mathrm{~min}^{-1}$.

spatially filtered with a $16.4 \times 16.4 \times 17.0 \mathrm{~mm}(7 \times 7 \times 4$ voxels) 3D boxcar filter. The time course of the radiotracer entering this transaxial slice can be seen in the large ROI time-activity curve in the graph on the left in Supplemental Figure 2. This plot illustrates that for regions containing significant spatial averaging, the SNR in the native FBP data and HYPR-LR processed data is almost equivalent. However, in the extreme case of a single voxel, the denoising properties of HYPR-LR processing are profound, as seen in Figure 6. In this limit, the SNR of each individual time point (for a single voxel) is strongly dependent on the SNR from the composite image, which is created by integrating the entire study (after the arrival of the radiotracer).

\section{DISCUSSION}

In this work, we have presented HYPR-LR processing in the context of dynamic PET as a tool for denoising individual time frames. The primary advantage of HYPR-LR postprocessing lies in its ease of implementation and fast processing time (approximately seconds per frame using a personal computer with a Pentium P4 [Intel] processor). To evaluate the accuracy of HYPR-LR processing and to examine the potential limits of the algorithm for yielding quantitative results, we chose a series of phantom, animal, and human studies with varying levels of noise and temporal kinetics.

\section{TABLE 1. ROI Estimates of Decay Constant $\left(\mathrm{min}^{-1}\right)$ \\ HYPR-LR

$\begin{array}{lccc}\text { Region } & \text { True value } & \text { FBP } & \begin{array}{c}(3 \times 3 \times 3 \text { voxels }) \\ \text { filter }\end{array} \\ { }^{11} \mathrm{C} & 0.0341 & 0.0347 \pm 0.0006 & 0.0345 \pm 0.0005 \\ { }^{18} \mathrm{~F} & 0.0069 & 0.0066 \pm 0.0005 & 0.0067 \pm 0.0001\end{array}$

Estimates were obtained using nonlinear least squares \pm SE.
We have not attempted to characterize a fixed metric for SNR improvement because this is highly dependent on the SNR in the composite image and the original images, which is dependent on the choice of time frame duration.

With HYPR-LR processing, the SNR of each time frame is primarily determined by the SNR in the composite image and the number of nonzero elements in the filtering function, $\mathrm{F}$ (18). The miniature Derenzo phantom was used to illustrate the improvement in SNR and preservation of resolution as determined by the composite image. Figure 2 demonstrates that the widths from the profiles, which are indicative of system resolution, are unchanged in the 2 image sets of FBP and HYPR-LR. Because the entire 60min image was used as the composite, this image largely determines the SNR and spatial resolution of the radioactivity distribution. The mean concentrations for the FBP and HYPR-LR data are almost equal, never exceeding a $2 \%$ difference between the methods and having a $0.15 \%$ mean difference over the entire profile. However, the variance of each point along the profile is reduced for the HYPR-LR data, yielding an improvement factor of 2.4 in SNR. Figure 2 illustrates that the variance at each point is also reduced using just the smoothing filter (by a factor of 1.7); however, this reduction is at the cost of reduced spatial resolution as seen by the inability to resolve the 1.5 -mm-diameter holes.

A multiradionuclide phantom was used to simulate a setting with varying kinetics over the course of a time series. Because the entire time series was used as the composite image (Fig. 3), the region with the ${ }^{11} \mathrm{C}$ activity appears to have concentration approximately equal to the surrounding ${ }^{18} \mathrm{~F}$ background activity. A range of spatial filters $(\mathrm{F})$ was applied to the composite and individual time frames to investigate bias in the measured signal. The effects of this spatial filtering were examined on both region-based and voxel-based time-activity curves. Increased spatial filtering produces significant improvements in SNR, which in turn 
FIGURE 5. HYPR-LR processing in study with rapid changes in PET signal. PET images represent single 2-min time frame (at 28 min after injection) of ${ }^{18} \mathrm{~F}$ fallypride in brain of rhesus monkey. This PET study consisted of 3 injections with radiolabeled and unlabeled fallypride at 0 (168 MBq, $4.2 \mathrm{nmol}), 20$ (189 MBq, $10.3 \mathrm{nmol})$, and $80 \mathrm{~min}(0$ $\mathrm{MBq}, 100 \mathrm{nmol})$, as indicated by arrows in graph. Graph represents time-activity curves from 4-voxel ROI with high and low radiotracer uptake, indicated by circles in bottom image.

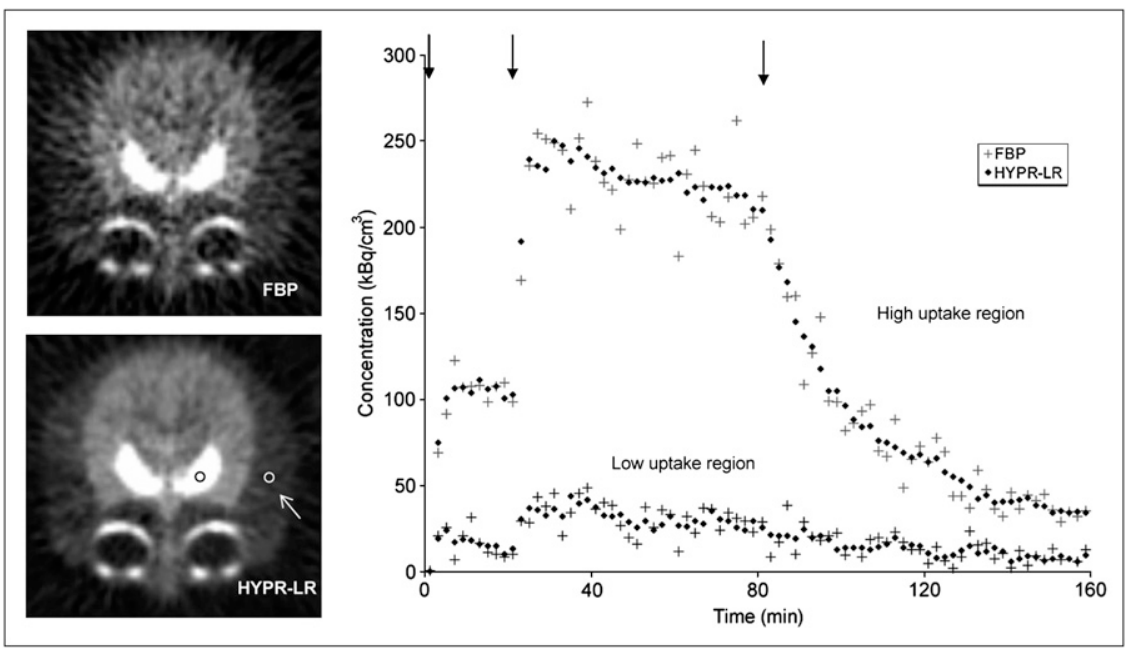

reduces the variance in the distribution of voxel decay constants. However, the excessive blurring in the signal from the surrounding regions introduces a bias in the decay constant outcome parameter (Supplemental Fig. 1). A bias of $21 \%$ was observed for $12.6 \times 12.6 \mathrm{~mm}(10 \times 10$ voxels $) 2 \mathrm{D}$ filtering and reduced to less than $2 \%$ for both $5.04 \times 5.04 \mathrm{~mm}$ $(4 \times 4$ voxels $) 2 \mathrm{D}$ and $3.78 \times 3.78 \times 3.63 \mathrm{~mm}(3 \times 3 \times 3$ voxels $)$ $3 \mathrm{D}$ filtering, which is well within the experimental error of PET measurements. These resolution effects were discussed for MRI applications by Johnson et al. (18) for considerations of filter size selection. As a general guideline, the effects of excessive blurring can be suppressed by choosing a filter that is approximately half the diameter of the object of interest.

Although HYPR-LR processing capitalizes on the spatiotemporal correlation existing in a PET time series, there is little temporal correlation introduced into the time series from HYPR-LR processing. The composite image contains information from all frames in a dynamic scan, but the noise components of each frame will be largely uncorrelated because of the long signal in the composite image with respect to the independent frames. Each time frame is calculated as the product of the composite (total sum) and a weighting matrix (Eq. 2), which contains only spatial smoothing. The time-activity curves from the multipleinjection study (Fig. 5) do not suggest that a temporal correlation was introduced in the HYPR-LR data. For example, the use of a moving average (time series) filter would tend to smooth out the transitions during the injections at 20 and $80 \mathrm{~min}$. Such trends were not observed in this dataset, which used the entire time series as the composite image.

Though we foresee several applications for which dynamic PET would benefit from HYPR-LR processing, we believe the greatest potential will be to improve quantitative PET for voxel-based analysis. For example, the Logan reference region method (24) is one of the most widely used schemes for the analysis of reversibly bound radiotracers; however, this method has been shown to demonstrate
FIGURE 6. HYPR-LR processing of ${ }^{15} \mathrm{O}$-water consisting of 60 frames with duration of $2 \mathrm{~s}$ each. Top image contains single 2-s frame of data from 26 to $28 \mathrm{~s}$. Bottom image contains matching frame after HYPR-LR processing $16.4 \times 16.4 \times$ $17.0 \mathrm{~mm}(7 \times 7 \times 4$ voxels $) 3 \mathrm{D}$ boxcar filter. Color bars are in units of $\mathrm{kBq} / \mathrm{mL}$. Graph illustrates time-activity curve of single voxel in region of arrow. (Supplemental Fig. 3 provides multiple images of time course.)

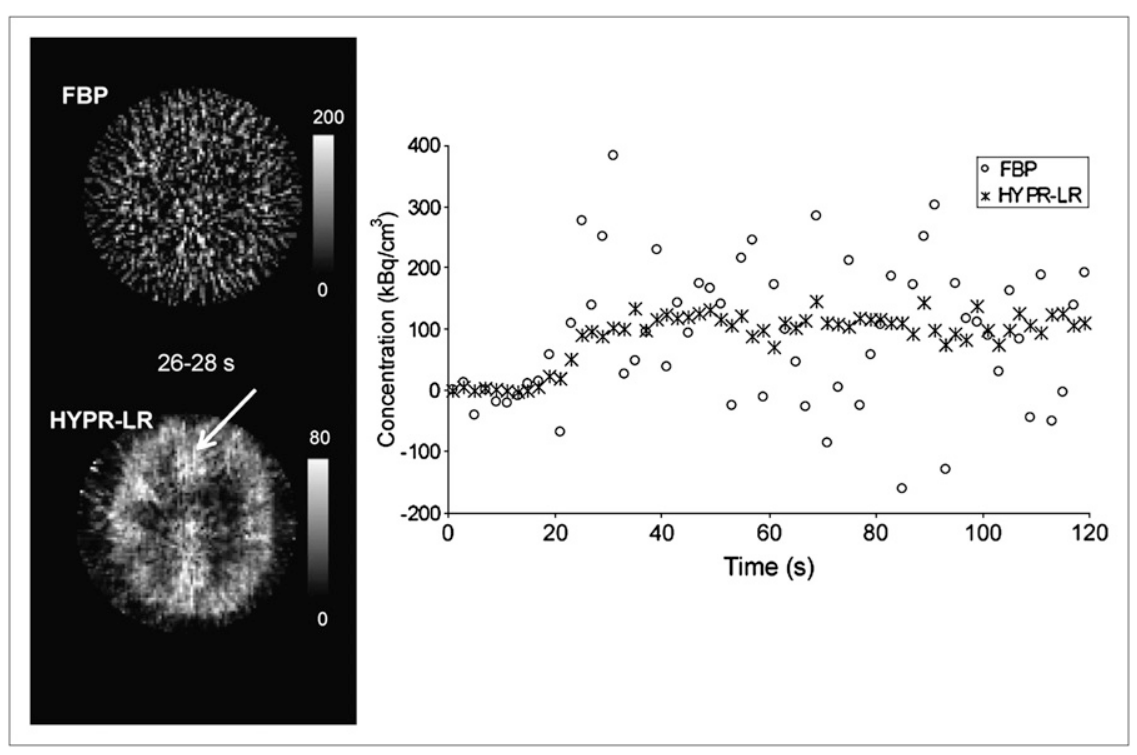


a negative bias in distribution volume ratio estimation with increasing levels of noise (25-28). This bias is due to the high correlation of noise in the independent and dependent variables, which are calculated directly from the PET data, and result in an underestimation of distribution volume ratio in the (higher noise) voxel-based estimates. Thus, for voxelbased comparisons of small regions, HYPR-LR processing holds the potential to enhance disease detection sensitivity or reduce subject sample size by improving parameter estimation accuracy. For example, the use of the Logan reference region method has been validated for the amyloid-binding radiotracer ${ }^{11} \mathrm{C}$-Pittsburgh compound B (29), which is being used to discriminate between subjects with Alzheimer disease and healthy controls in the Alzheimer Disease Neuroimaging Initiative multicenter trial, and HYPR-LR may enhance the sensitivity of studies with this radiotracer. A similar application is presented with the ${ }^{15} \mathrm{O}$-water study. Although cerebral perfusion (or oxygen metabolism) estimation with HYPR-LR processing will not be improved with analysis techniques that integrate the entire PET time course-for example, a count-based method (30)_HYPRLR processing does open up opportunities for improving the sensitivity using other voxel-based methods of analysis.

Other examples include studies with ${ }^{11} \mathrm{C}$-WAY100635, in which injections are limited to $260 \mathrm{MBq}$ (31) but scans in excess of 90 are required, and research protocols involving children, for whom only $10 \%$ of the radiation dose of comparable adult studies is allowed. Similarly, PET methods that rely on the measurement of subtle changes in the dynamic PET signal for detecting neurotransmitter release in small regions (32-37) may benefit profoundly from HYPR-LR denoising. Most important, as data acquisition systems for PET continue to improve, it may be possible to develop clinical applications that rely on visual image (or time series) interpretation (2), which benefits significantly from HYPR-LR processing.

\section{CONCLUSION}

In this work, we presented the application of HYPR-LR for denoising PET data for time series analysis. This method has potential applications in imaging protocols with an inherently low SNR that is due either to an extended dynamic acquisition duration or a low radiation dose.

\section{ACKNOWLEDGMENTS}

We thank Drs. Miguel Alivia-Rodriguez (UNAM) and John Nye (Emory University) for the use of the phantom, Dr. Alex Converse and Elizabeth Ahlers for technical assistance with the microPET scanner, Barb Mueller for the acquisition of the human PET studies, and Dr. Dana Tudorascu for technical discussions. This study was presented in part at the 54th annual Society of Nuclear Medicine Meeting, Washington, DC, June 2007.

\section{REFERENCES}

1. Phelps ME. Positron computed tomography studies of cerebral glucose metabolism in man: theory and application in nuclear medicine. Semin Nucl Med. 1981;11:32-49.

2. Di Chiro G, Brooks RA. PET quantitation: blessing and curse. J Nucl Med. 1988; 29:1603-1604.

3. Keyes JW. SUV: standard uptake or silly useless value. J Nucl Med. 1995;36: 1836-1839.

4. Hamberg LM, Hunter GJ, Alpert NM, Choi NC, Babich JW, Fischman AJ. The dose uptake ratio as an index of glucose-metabolism: useful parameter or oversimplification. J Nucl Med. 1994;35:1308-1312.

5. Huang SC. Anatomy of SUV. Nucl Med Biol. 2000;27:643-646.

6. Young H, Baum R, Cremerius U, et al. Measurement of clinical and subclinical tumour response using [F-18]-fluorodeoxyglucose and positron emission tomography: review and 1999 EORTC recommendations. Eur J Cancer. 1999; 35:1773-1782.

7. Carson RE, Lange K. A statistical model for positron emission tomography: the EM parametric image reconstruction algorithm-comment. J Am Stat Assoc. 1985;80:20-22.

8. Snyder DL. Parameter estimation for dynamic studies in emission-tomography systems having list-mode data. IEEE Trans Nucl Sci. 1984;31:925-931.

9. Ollinger JM. Estimation algorithms for dynamic tracer studies using positronemission tomography. IEEE Trans Med Imaging. 1987;6:115-125.

10. Kao CM, Yap JT, Mukherjee J, Wernick MN. Image reconstruction for dynamic PET based on low-order approximation and restoration of the sinogram. IEEE Trans Med Imaging. 1997;16:738-749.

11. Wernick MN, Infusino EJ, Milosevic M. Fast spatio-temporal image reconstruction for dynamic PET. IEEE Trans Med Imaging. 1999;18:185-195.

12. Millet P, Ibanez V, Delforge J, Pappata S, Guimon J. Wavelet analysis of dynamic PET data: application to the parametric imaging of benzodiazepine receptor concentration. Neuroimage. 2000;11:458-472.

13. Turkheimer FE, Banati RB, Visvikis D, Aston JAD, Gunn RN, Cunningham VJ. Modeling dynamic PET-SPECT studies in the wavelet domain. J Cereb Blood Flow Metab. 2000;20:879-893.

14. Cselenyi Z, Olsson H, Farde L, Gulyas B. Wavelet-aided parametric mapping of cerebral dopamine D-2 receptors using the high affinity PET radioligand [C11]FLB 457. Neuroimage. 2002;17:47-60.

15. Alpert NM, Reilhac A, Chio TC, Selesnick I. Optimization of dynamic measurement of receptor kinetics by wavelet denoising. Neuroimage. 2006;30: $444-451$.

16. Zhou Y, Huang SC, Bergsneider M, Wong DF. Improved parametric image generation using spatial-temporal analysis of dynamic PET studies. Neuroimage. 2002;15:697-707.

17. Mistretta CA, Wieben O, Velikina J, et al. Highly constrained backprojection for time-resolved MRI. Magn Reson Med. 2006;55:30-40.

18. Johnson KM, Velikina J, Wu Y, Kecskemeti S, Wieben O, Mistretta CA. Improved waveform fidelity using local HYPR reconstruction (HYPR LR). Magn Reson Med. 2008;59:456-462.

19. Boussion N, Hatt M, Lamare F, et al. A multiresolution image based approach for correction of partial volume effects in emission tomography. Phys Med Biol. 2006;51:1857-1876.

20. Tai C, Chatziioannou A, Siegel S, et al. Performance evaluation of the microPET P4: a PET system dedicated to animal imaging. Phys Med Biol. 2001;46:18451862 .

21. Tai YC, Ruangma A, Rowland D, et al. Performance evaluation of the microPET Focus: a third-generation microPET scanner dedicated to animal imaging. $\mathrm{J} \mathrm{Nucl}$ Med. 2005;46:455-463.

22. Avila-Rodriguez MA. Low Energy Cyclotron Production of Multivalent Transition Metals for PET Imaging and Therapy. Madison, WI: Medical Physics, University of Wisconsin-Madison; 2007.

23. Christian BT, Narayanan T, Shi B, Morris ED, Mantil J, Mukherjee J. Measuring the in vivo binding parameters of [F-18]-fallypride in monkeys using a PET multiple-injection protocol. J Cereb Blood Flow Metab. 2004;24:309-322.

24. Logan J, Fowler JS, Volkow ND, Wang GJ, Ding YS, Alexoff DL. Distribution volume ratios without blood sampling from graphical analysis of PET data. J Cereb Blood Flow Metab. 1996;16:834-840.

25. Carson RE. PET parameter estimation using linear regression methods: bias and variability considerations. In: Uemura K, Lassen NA, Jones T, Kanno I, eds. Quantification of Brain Function: Tracer Kinetics and Image Analysis in Brain PET. Amsterdam, The Netherlands: Elsevier; 1993:499-507.

26. Hsu B, Alpert NM, Christian BT, Bonab AA, Morris ED, Fischman AJ. Noise properties of a graphical assay of receptor binding [abstract]. J Nucl Med. 1997; 38:204P. 
27. Slifstein M, Laruelle M. Effects of statistical noise on graphic analysis of PET neuroreceptor studies. J Nucl Med. 2000;41:2083-2088.

28. Logan J, Fowler JS, Volkow ND, Ding YS, Wang GJ, Alexoff DL. A strategy for removing the bias in the graphical analysis method. J Cereb Blood Flow Metab. 2001;21:307-320.

29. Price JC, Klunk WE, Lopresti BJ, et al. Kinetic modeling of amyloid binding in humans using PET imaging and Pittsburgh compound-B. J Cereb Blood Flow Metab. 2005;25:1528-1547.

30. Derdeyn CP, Videen TO, Simmons NR, et al. Count-based PET method for predicting ischemic stroke in patients with symptomatic carotid arterial occlusion. Radiology. 1999;212:499-506.

31. Parsey RV, Belanger MJ, Sullivan GM, et al. Biodistribution and radiation dosimetry of C-11-WAY100,635 in humans. J Nucl Med. 2005;46:614-619.

32. Aston JAD, Gunn RN, Worsley KJ, Ma Y, Evans AC, Dagher A. A statistical method for the analysis of positron emission tomography neuroreceptor ligand data. Neuroimage. 2000;12:245-256.
33. Friston KJ, Malizia AL, Wilson S, Cunningham VJ, Jones T, Nutt DJ. Analysis of dynamic radioligand displacement or "activation" studies. J Cereb Blood Flow Metab. 1997;17:80-93.

34. Pappata S, Dehaene S, Poline JB, et al. In vivo detection of striatal dopamine release during reward: a PET study with $\mathrm{C}-11$ raclopride and a single dynamic scan approach. Neuroimage. 2002;16:1015-1027.

35. Alpert NM, Badgaiyan RD, Livni E, Fischman AJ. A novel method for noninvasive detection of neuromodulatory changes in specific neurotransmitter systems. Neuroimage. 2003;19:1049-1060.

36. Christian BT, Lehrer DS, Shi BZ, et al. Measuring dopamine neuromodulation in the thalamus: using F-18 fallypride PET to study dopamine release during a spatial attention task. Neuroimage. 2006;31:139-152.

37. Normandin MD, Morris ED. Estimating neurotransmitter kinetics with ntPET: a simulation study of temporal precision and effects of biased data. Neuroimage. 2008;39:1162-1179. 